\title{
Freshness assessment of tilapia fish in traditional market based on an electronic nose
}

\author{
Radi, Eka Wahyudi, Muhammad Danu Adhityamurti, Joko Purwo Leksono Yuroto Putro, Barokah, \\ Dwi Noor Rohmah \\ Department of Agricultural and Biosystems Engineering, Faculty of Agricultural Technology, Universitas Gadjah Mada,
} Yogyakarta

\section{Article Info}

Article history:

Received Aug 31, 2020

Revised May 20, 2021

Accepted Jul 24, 2021

\section{Keywords:}

Ammonia test kit

Aroma pattern

Electronic nose

Freshness level

Neural network

Sensor array

Tilapia

\begin{abstract}
This study evaluates an e-nose based on gas sensors to measure the freshness of tilapia. The device consists of a series of semiconductor sensors as detector, a combination of valve-vial-oxygen as sample delivery system, a microcontroller as interface and controller, and a computer for data recording and processing. The e-nose was firstly used to classify the fresh and non-fresh tilapia. A total of 48 samples of fresh tilapia and 50 samples of non-fresh tilapia were prepared and measured using the e-nose through three stages, namely: flushing, collecting, and purging. The sensor responses were processed into aroma patterns, then classified by two pattern classification softwares of principal component analysis (PCA) and neural network (NN). There were four methods for aroma patterns formation being evaluated: absolute data, normalized absolute data, relative data, normalized relative data. The results showed that the normalized absolute data method provides the best classification with the accuracy level of $93.88 \%$. With this method, the trained $\mathrm{NN}$ was used to predict the freshness of 15 tilapia samples collected from a traditional market. The result showed that $60.0 \%$ of the samples are classified into fresh category, $33.3 \%$ are in the non-fresh category, and $6.7 \%$ are not included in both categories.
\end{abstract}

This is an open access article under the CC BY-SA license.

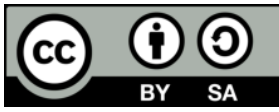

\section{Corresponding Author:}

Radi

Department of Agricultural and Biosystems Engineering

Faculty of Agricultural Technology

Universitas Gadjah Mada

Jl. Flora no. 1, Bulaksumur, Yogyakarta 55281, Indonesia

Email: radi-tep@ugm.ac.id

\section{INTRODUCTION}

Fresh fish is one of the food products that is easily damaged (rotten) if the product is not treated properly. Generally, after the fish are caught or harvested, a series of quality degradation processes in the form of reactions that reshuffle its compounds will occur. Fish products have a high fluid content and excellent nutrition for the growth of destructive bacteria [1]. These bacteria will accelerate the decay of fish products. In general, there are three processes that cause the decrease of fish products quality, namely autolysis, bacteriological, and chemical processes. There are several treatments that need to be conducted in order to slow down the progress of these processes, so the freshness level of fish products can be maintained in a good condition for a longer time.

Storage technology for fresh fish today may have been developed. However, fish traders in traditional markets are generally not familiar with and never apply this technology due to cost considerations. The common technology of storage at low temperatures may reduce the product spoilage rate. However, this 
treatment requires additional equipment such as a refrigerator or ice blocks. This treatment certainly has an impact on the products marketing costs. This kind of treatment is rarely carried out by fish traders, especially those who trade in traditional markets. As a product that is easily damaged, this condition makes fresh fish products sold in traditional markets have a high potential for spoilage. Thus, freshness assessment for the fish product sold at traditional is needed. One of the fresh fish commodities is tilapia.

Tilapia is a type of freshwater fish that is widely cultivated and has high economic potential. The demand and supply of tilapia on the international market has shown a significant increase since 2015 [2]. Besides it is delicious, rich in nutrients such as protein, vitamins, minerals needed by the human body, tilapia is also easy to cultivate, that makes its production cost relatively low, drives it to have a great demand by consumers, and is widely traded in traditional markets, including in the region of Yogyakarta. However, the quality of tilapia declines very quickly after being harvested if not handled properly. Naturally, tilapia will experience quality degradation after 8-10 h from being harvested which is marked by the formation of an unpleasant aroma released by the fish [3]. The freshness of tilapia can be maintained maximum $12 \mathrm{~h}$ at ambient temperature after being harvested and up to 15 days through low temperature treatment [4]. Based on the number of tilapias that are traded after being slaughtered at the traditional market, a research of the commodities freshness level needs to be done.

Research of the fish freshness level has been carried out by many researchers. Generally, the study of fish freshness is carried out with several quality parameters such as sensory, chemical, physical, and microbiological [5]. The human olfactory system is one of the natural tools that can be used to assess the quality of fish products, however, this assessment often produces biased results, and the human olfactory system does not have the ability to detect toxic gases [6]. Research shows that parameter of $\mathrm{pH}$ has a relationship to the level of tilapia freshness [7]. Recently, research of fish freshness has focused on developing quality measuring devices that are fast, portable, and inexpensive. There are many types of alternative devices that were created to replace natural assessment tools such as image processing devices [2] and electronic nose [8]. Electronic nose (e-nose) is one of the devices developed to identify the scent of the sample.

Since aroma still becomes the main parameter in food products quality control, the use of aroma meters such as an e-nose will increase in the future, including in the determination of fish products quality. Currently e-nose is widely used in various fields such as quality control of food products [9-10], assessment and classification of meat freshness [11]-[13], determination of fish freshness [8], classification of fruits [14], characterization of tea aroma [15]-[16], identification and classification of coffee aroma [17]-[25], identification of environmental quality [26], and medical purposes such as identification of respiratory disease [27]. E-nose produces a specific response when a sample of aroma compound is exposed on the sensor array headspace. These responses are then converted into a specific pattern that can be recognized by pattern recognition methods such as principal component analysis (PCA) [17], [20], [28], common dimension analysis (ComDim) [24], linear discriminant analysis (LDA) [24] and multiple discriminant analysis (MDA) [29] or other pattern recognition methods.

The aroma of fish products is one of the quality parameters that has the potency to be used to determine the level of freshness. According to [5], study about e-nose as an assessment device for fish freshness has been carried out, but the research is only limited for sardine products. The result shows that the e-nose has some advantages for the fish product, such as fast assessing process and accurate results.

\section{METHOD}

\subsection{Material}

This study evaluates the application of an e-nose in determining the freshness level of tilapia traded in traditional markets in Yogyakarta. The study started with the classification of fresh and non-fresh tilapia by using the e-nose. There were three categories of samples used: a) fresh tilapia; b) non-fresh tilapia; and c) tilapia randomly obtained from fish traders. Samples A and B were prepared by buying live tilapia on the market, brought it to the laboratory and then slaughtered. Experiment with samples A was carried out within a maximum duration of 1 hour after being slaughtered, while for samples B was carried out after being stored for 10-24 $\mathrm{h}$ at laboratory without treatment (according to [8]). Samples $\mathrm{C}$ were prepared by buying slaughtered tilapia from fish traders in traditional markets. In addition, this study also used ammonia test kits to assess the freshness of tilapia. Samples A consisted of 48 samples, samples B consisted of 50 samples, and samples $\mathrm{C}$ consisted of 15 samples. Each sample assessment by using e-nose was carried out by using $7 \mathrm{~g}$ of tilapia sample piece.

\subsection{Design of electronic nose}

E-nose used in this study consisted of four main parts, namely an odor handling and delivery system, a detector, a signal conditioning system, and a data acquisition device [20]. The odor handling and delivery system consisted of two valves to regulate the circulation of aroma samples and carrier gas 
(oxygen). The detector consisted of 12 semiconductor gas sensors that are connected to the resistor in series based on the voltage divider principle. The microcontroller was used for controlling the entire e-nose components and also used to convert the analog signal from the sensor into a digital signal (as analog digital converter, ADC). Beside the interface to control the entire system during the sample handling process, the data acquisition device was also functioned to collect, display, and store all data. Besides, the e-nose was also equipped with a temperature-humidity sensor and a flow meter. The e-nose design is presented in Figure 1.

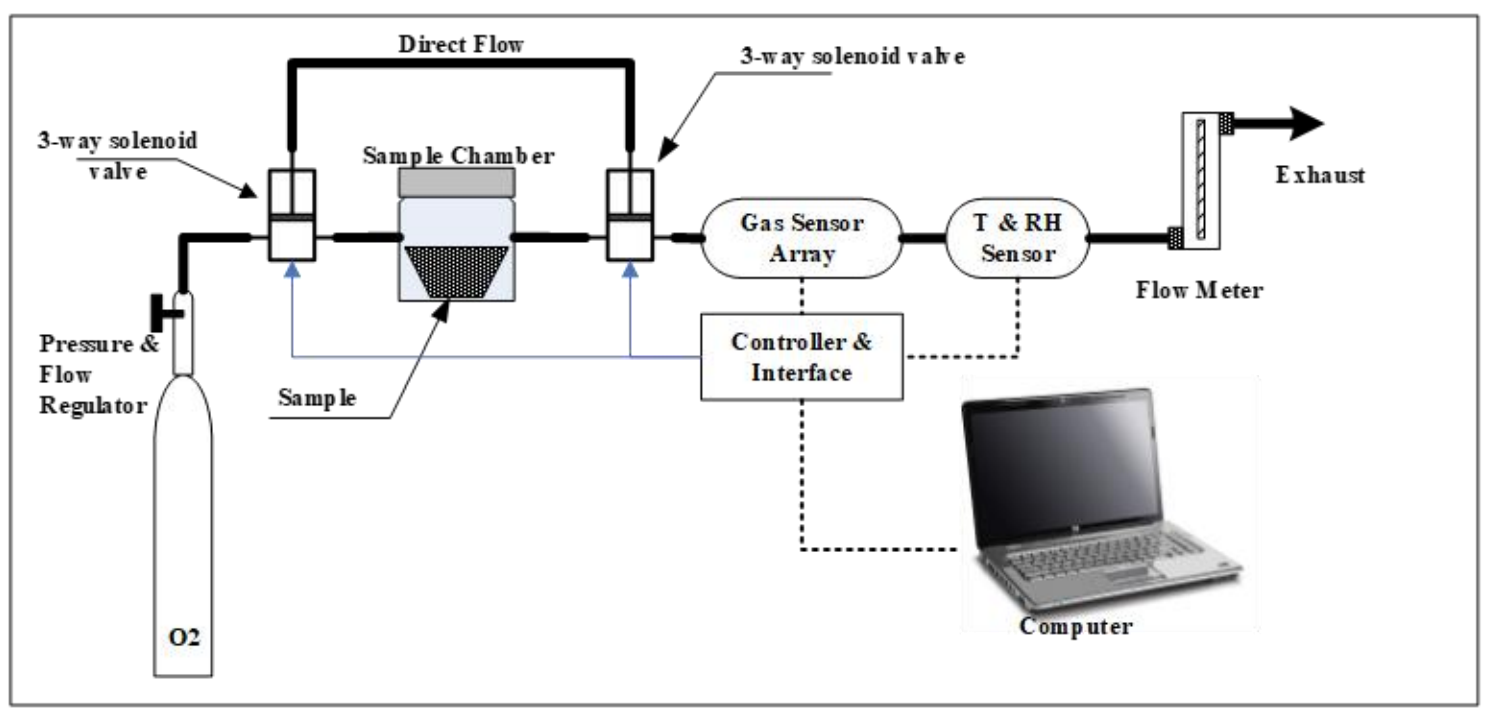

Figure 1. The design of the e-nose

\subsection{Experimental procedure}

\subsubsection{Sample preparation}

Each category of samples was prepared to be pieces of fish with a uniform weight of $7 \mathrm{~g}$. Each sample was prepared by being put in a cup inside the sample chamber, then allowed to stand for 3 minutes inside while the sample chamber closed tightly. After that, data collection could be started.

\subsubsection{Electronic nose preparation}

Before being used for collecting sample data, it must be ensured that all components of the e-nose were available and could be operated properly. Flow rate of the carrier gas was set to be constant at 1 $\mathrm{mL} / \mathrm{min}$. The e-nose was connected to a personal computer (PC) or a laptop as a data acquisition device, then turned it on. The e-nose was preheated by selected cleaning mode on the prepared graphical user Interface (GUI) of the data acquisition program that is installed in the connected PC or laptop. During one cycle of cleaning process ( 5 minutes), trying the e-nose was conducted by operating it without a sample. During the trial, the sensor's response was recorded and displayed. Stable sensors' response indicated that the e-nose was ready to be used for measurement.

\subsubsection{Data collection}

Data collection was carried out after samples and e-nose preparation. Data collection was started by pressing the start button on the data acquisition programs. Data collection was carried out through 3 stages, namely flushing, collecting, and purging. Each stage took two, three, and two minutes, respectively. Thus, the measurement with the e-nose needed a total time of 10 minutes per sample ( 3 minutes of sample preparation and 7 minutes of data collection). The response data of the sensor array along with the humidity and temperature data of each sample was recorded and stored in a connected PC or laptop for further processing.

\subsubsection{Data analysis}

Data analysis was started with the formation of sample aroma patterns from the collected sensor response data. The aroma pattern of the sample was formed from the response of the gas sensor series of each analyzed sample. In forming the aroma pattern, this study evaluated four methods, namely 1) absolute data; 2) normalized absolute data; 3) relative data; 4) normalized relative data. Each pattern formation method is shown in (1)-(4): 


$$
\begin{aligned}
A_{i} & =\frac{\sum_{t=291}^{300} x_{i, t}}{10} \\
B_{i} & =\frac{A_{i}}{\max A_{i}} \\
C_{i} & =\frac{\sum_{t=291}^{300} x_{i, t}-\sum_{t=111}^{120} x_{i, t}}{10} \\
D_{i} & =\frac{C_{i}}{\max C_{i}}
\end{aligned}
$$

Where A, B, C, and D are aroma patterns formed by the method of absolute data, normalized absolute data, relative data, and normalized absolute data; $x_{i, t}$ is the response of the $\mathrm{i}^{\text {th }}$ sensor as a function of time $\mathrm{t}(\mathrm{s}) ; \mathrm{i}$ is the sensor index. An illustration of the pattern formation method is presented in Figure 2. The patterns are then depicted on a radar graph to visualize the differences between the aroma patterns formed.

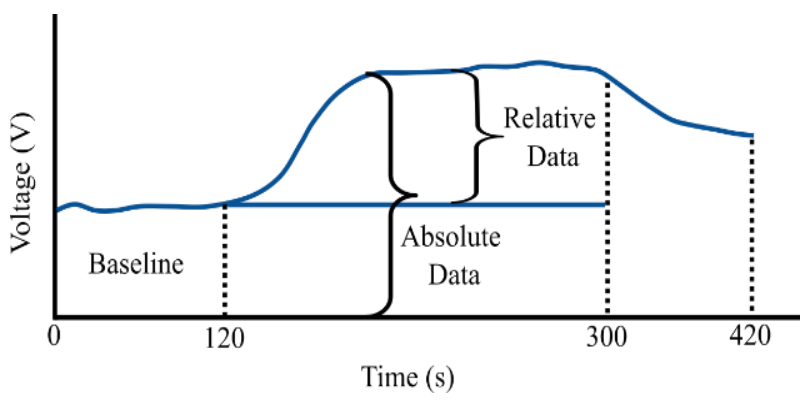

Figure 2. Illustration of the formation method of aroma pattern

Each formed sample's aroma patterns were then analyzed in order to get its distribution. This analysis was held by using PCA and neural network (NN) analysis. PCA was used to represent pattern separation linearly, while the NN analysis was applied to represent the non-linear one. The result of PCA was the distribution of aroma patterns that can be analyzed visually and makes data interpretation easier. Meanwhile, analysis with the NN will facilitate its implementation. PCA is an effective unsupervised multivariate analysis that can be applied to transform data with high-dimension (many variables) into lowdimensional spaces without losing data characteristics. PCA commonly be used in various analyses, such as for data analysis on an e-nose device [29] and also be used in the same research on image processing [30]. An illustration of the PCA analysis when applied on an e-nose is presented in [20].

\section{RESULTS AND DISCUSSION}

\subsection{Sensor response}

When data collection began, carrier gas would flow to the detector chamber directly as the flushing process was going on. During this process, the sensor array was cleaned from previous samples aroma exposure. Sensor array produced response that will be used as a reference. After two minutes of flushing, the microcontroller would open the valve in order to flow carrier gas through the sample chamber. Thus, sample aroma consisted of various volatile compounds from the samples that could flow together with carrier gas and be exposed to the sensor array in the detector chamber. Exposure of samples aroma was then responded to by the sensor array. The response is in the form of a change in sensor resistance which is then converted into a voltage shift. Collecting process ran for 3 minutes, then followed by closing the valve indicated for starting the purging. The purging process would flow the carrier gas directly to the detector chamber again in order to clean the sensor array from previous exposure of the sample. The process lasted for 2 minutes. An example of one cycle data collection is presented in Figure 3. This response consistent with another research [3]. Based on these data, the sensor response to samples aroma exposure was indicated by a voltage increment of $50-250 \mathrm{mV}$.

The e-nose used in this study was equipped with temperature and humidity sensors that were mounted on the carrier gas channel outside the detector chamber, thus after flowing through the detector chamber, the carrier gas flowed through the temperature and humidity sensor. The response of the 
temperature and humidity sensor was used to describe the temperature and humidity of the mixture of carrier gas and sample. It was also applied to indicate whether the sampling process was going well or not. The example of one cycle temperature and humidity sensor response during data collection is presented in Figure 4, shows that the sample temperature was ranged at $28-30^{\circ} \mathrm{C}$, while the humidity was ranged at $60-70 \%$.

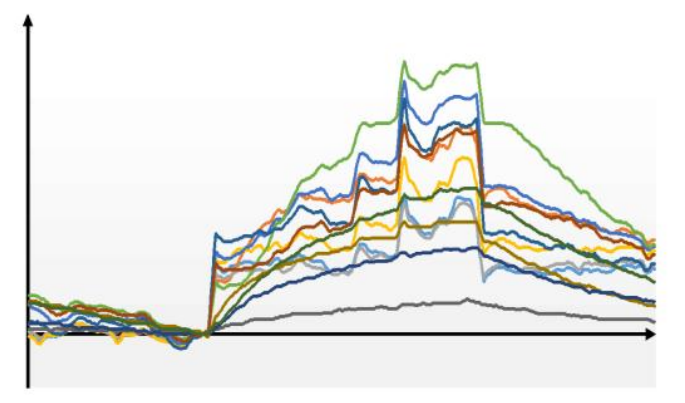

Figure 3. Response of sensor array with the presentation of fresh sample

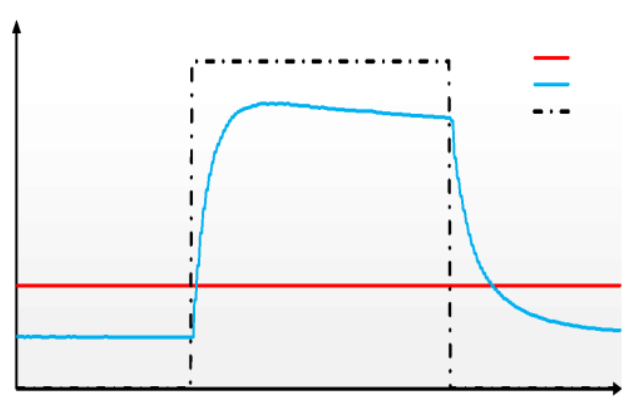

Figure 4. Temperature and humidity of the presentation of fresh sample

\subsection{Aroma pattern of fresh and non-fresh sample}

Data analysis was started by forming aroma patterns from the fresh sample (A) and non-fresh samples (B). The patterns were represented on radar graphs. Aroma patterns formed by all four pattern formation methods are presented in Table 1. These patterns show that absolute data (a) and normalized absolute data method (b) produce consistent patterns between samples in the same group, while relative data (c) and normalized relative data method (d) produce deviant patterns of some repetitions data. Visually, the pattern formed by all these methods for fresh and non-fresh samples tended to be similar one to another. Thus, visual classification based on these patterns could not be done and analysis based on pattern recognition algorithms needed to be carried out.

Table 1. Aroma pattern of fresh sample (A) and non-fresh sample (B) with different formation method

Method for Aroma Pattern Formation




\subsection{PCA method to classify the fresh and non-fresh sample}

PCA is a transformation method of multi-variable data, in which they may interact with each other, into new data variables with new coordinates that are not mutually correlated, called the principal component (PC). In PCA, new variables are ordered based on the number of data variance which may be associated with the carried information content. Usually, the use of two or three PCs can represent the whole information contained on the data. With these two or three variables, sample aroma patterns can be visualized in Cartesian coordinates in either $2 \mathrm{D}$ or $3 \mathrm{D}$. The distribution of aroma patterns of samples A and $\mathrm{B}$ which were analyzed by using the PCA method is presented in Figure 5. The graph illustrates the distribution of sample aroma patterns formed by all the pattern formation methods. Green square symbol represents the fresh sample (A) while red square symbol represents the non-fresh sample.

Figure 5 (a) is the result of PCA analysis of the samples aroma patterns formed by absolute data method (a). The PC- 1 represents $80.8 \%$ of the variance of the data, while the PC- 2 carries $11 \%$. Thus, Figure 5 (a) has represented $91.8 \%$ information from the whole data. Figure 5 (b) is the result of PCA analysis for sample aroma patterns formed by normalized absolute data method (b) with an accumulation of information of $83.9 \%$ (PC-1 is $66.8 \%$ and PC-2 is $17.1 \%$ ). Figure 5 (c) is PCA analysis for samples aroma patterns formed by the relative data method that carries accumulated information of $89.4 \%$ (PC-1 is $65.4 \%$ and PC-2 is 24\%). Figure 5 (d) is the result of PCA analysis for samples aroma patterns formed by normalized relative data method (d) that represents $99.61 \%$ information from the whole data (PC-1 is $99.1 \%$ and PC-2 is $0.51 \%$ ). Method of $\mathrm{b}$ shows a more separable distribution between samples A and B. In other words, method $\mathrm{b}$ provides a data distribution that is easier to classify than the other methods.

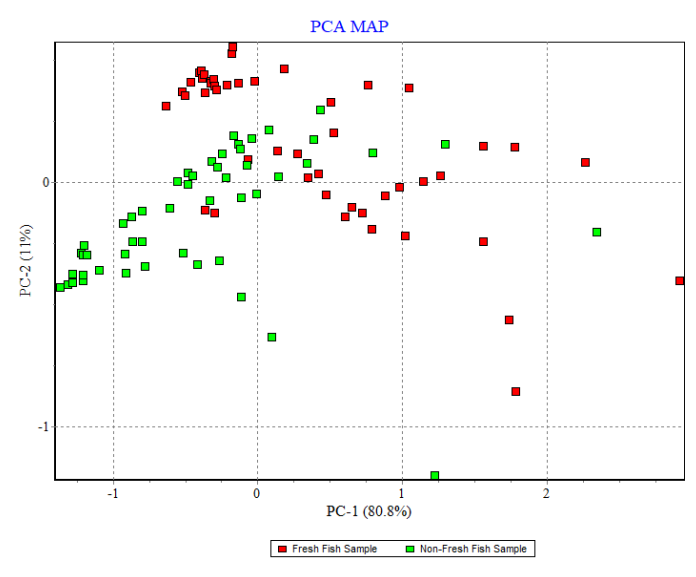

(a)

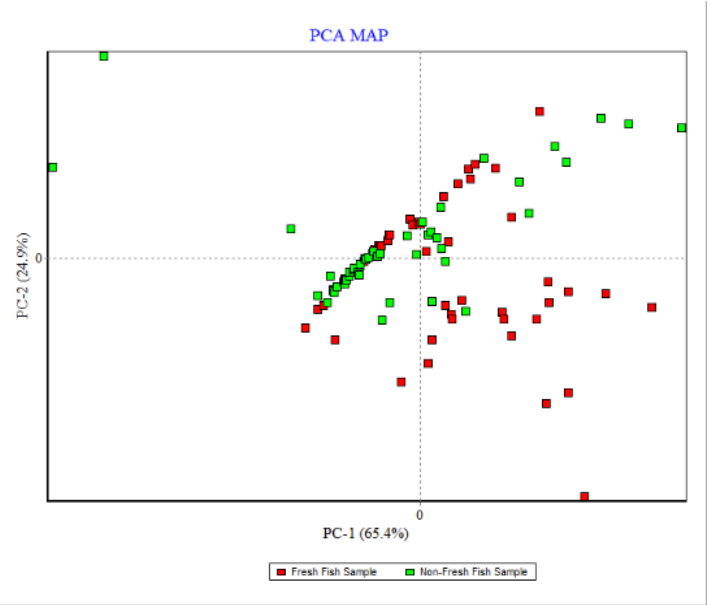

(c)

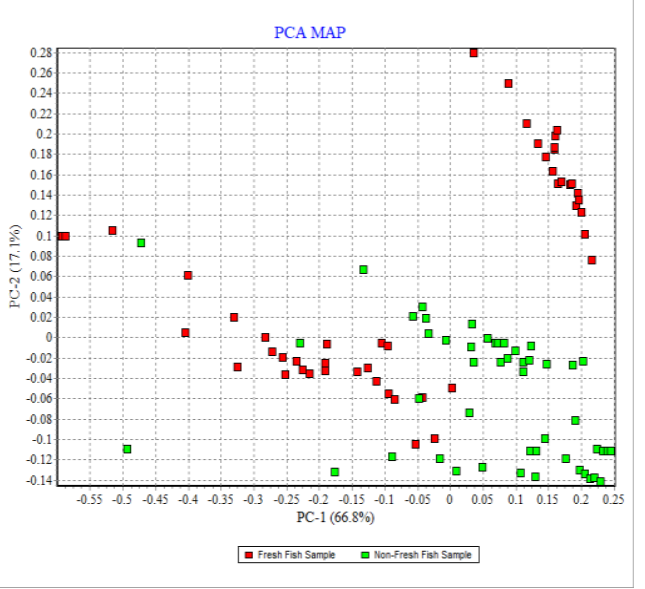

(b)

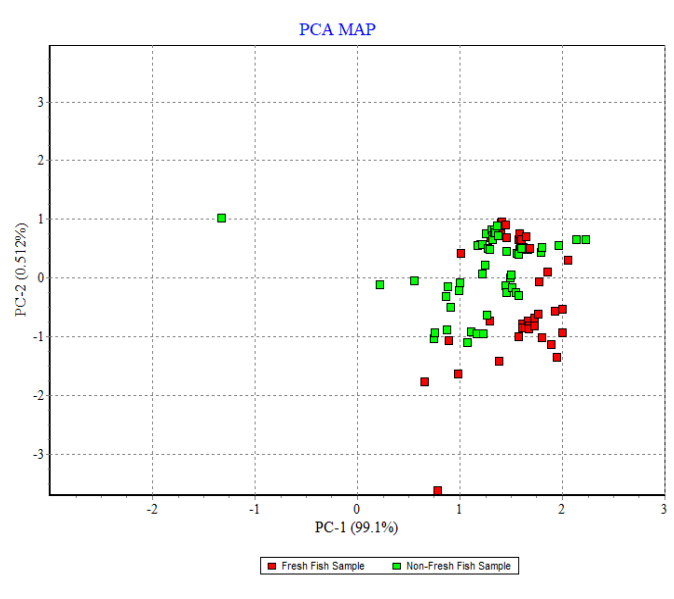

(d)

Figure 5. Scatter plot of PCA for fresh and non-fresh sample with different preprocessing; (a) absolute data, (b) normalized absolute data, (c) relative data, (d) normalized relative data 


\subsection{Classification of fresh and non-fresh sample with neural network}

PCA results provided data distribution that could be visually analyzed to identify the pattern separation of the samples. However, the PCA result did not provide quantitative information about the level of the pattern classification. In order to measure the separation level of the samples quantitatively, the analysis was then continued by using a NN. The NN was designed with a layer structure of 12-50-20-2 (12 nodes of input corresponds to the number of sensors being applied, 50 nodes of first hidden layer, 20 nodes of second hidden layer, and 2 nodes of output, corresponding to the two groups of samples being separated). The NN was then trained to recognize the training data set by using an error back propagation training method with the learning rate of 0.250 , the momentum of 0.1 , and the iteration number of 10000 . The amount of training data set was varied by $100 \%, 75 \%, 50 \%, 25 \%$, and $10 \%$ of the number of the collected data. After being trained, the NN was used to classify the test data which consists of all formed patterns. Pattern formed by absolute data method (a) provides classification accuracy ranged at 73-100\%. Meanwhile, by using the result of the normalized absolute data method (b), the $\mathrm{NN}$ is able to classify the samples with the accuracy range of $87-100 \%$. The NN trained with sample aroma pattern formed by relative data method (c) produces a classification accuracy ranging at $48-98 \%$, and classification accuracy that ranged at $57-98 \%$ obtained by the pattern formation method of normalized absolute data (d). Based on these results, normalized absolute data method (b) produces the highest accuracy of classification with the average of $93.88 \%$. Thus, this method could be applied as a pattern formation method in the further development of the e-nose, especially for the determination of tilapia products' freshness. The classification accuracy of the NN with varied aroma pattern formation method and varied number of training data set for fresh and non-fresh samples are presented in Table 2.

Table 2. The results of the neural network analysis for the classification of aroma patterns of fresh tilapia and

\begin{tabular}{cccccc}
\multicolumn{6}{c}{ non-fresh tilapia samples using four preprocessing methods } \\
\hline $\begin{array}{c}\text { Method for Aroma } \\
\text { Pattern Formation }\end{array}$ & $\begin{array}{c}\text { Number of Training } \\
\text { Data Set }\end{array}$ & $\begin{array}{c}\text { Number of } \\
\text { Testing Data }\end{array}$ & $\begin{array}{c}\text { Number of } \\
\text { Correct }\end{array}$ & $\begin{array}{c}\text { Accuracy } \\
(\%)\end{array}$ & $\begin{array}{c}\text { Accuracy in } \\
\text { Average (\%) }\end{array}$ \\
\hline Absolute Data & 98 & 98 & 98 & 100.00 & 86.53 \\
& 76 & 98 & 98 & 100.00 & \\
& 50 & 98 & 80 & 81.63 & \\
Normalized & 26 & 98 & 72 & 73.47 & \\
Absolute Data & 10 & 98 & 76 & 77.55 & \multirow{2}{*}{93.88} \\
& 98 & 98 & 98 & 100.00 & \\
Relative Data & 76 & 98 & 98 & 100.00 & \\
& 50 & 98 & 89 & 90.82 & \\
& 26 & 98 & 89 & 90.82 & \\
& 10 & 98 & 86 & 87.76 & \\
Normalized Relative & 98 & 98 & 97 & 98.98 & \\
Data & 56 & 98 & 96 & 97.96 & \\
& 26 & 98 & 68 & 74.49 & \\
& 10 & 98 & 48 & 48.39 & \\
& 98 & 98 & 97 & 98.98 & 79.18 \\
& 50 & 98 & 96 & 97.96 & \\
& 26 & 98 & 69 & 70.41 & \\
& 10 & 98 & 70 & 71.43 & \\
\hline
\end{tabular}

\subsection{Freshness identification of tilapia from traditional market}

Identification of the freshness level of tilapia obtained randomly from traditional market traders was done by comparing its aroma patterns to previous patterns that have been collected and analyzed (patterns of samples A and B). There were 15 fish samples (C) to be analyzed. Data collection process was carried out in exactly the same way as the previous data collection. The response of the sensor array was converted into an aroma pattern by using the normalized absolute data method (b). Sample aroma pattern formed is presented in Figure 6, shows that the aroma patterns of the samples appear to vary. Sample aroma pattern was then analyzed by using the trained NN (trained with all data of fresh and non-fresh sample). The results of the classification for these samples is presented in Figure 7. This result shows that $60.0 \%$ of the samples were match to the fresh category, $33.3 \%$ were match to the non-fresh category, and $6.7 \%$ were not match both categories. 


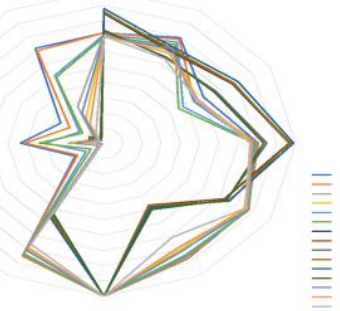

Figure 6. Aroma pattern of tilapia sample from traditional market
Classification of Sample (\%)

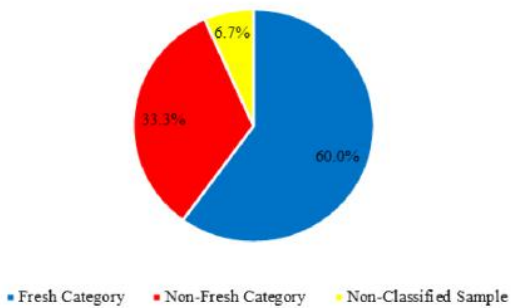

Figure 7. Freshness identification of tilapia sample from traditional market

\subsection{Analysis with ammonia and formalin test kit}

The spoilage process of fish products after being harvested at room temperature, usually occurs due to autolysis, bacteriological, and chemical processes [1], [4], [31]. The degradation process is characterized by the appearance of mucus, meat softening, and the appearance of unpleasant aromas [31]. Ammonia gas that contributes to that unpleasant aroma, generally appears along with the increase of spoilage level of the fish. Recent research shows that the ammonia compounds are also used for the freshness indicator of the packed fish [32]. In order to see the difference of ammonia content of samples A, B, and C, they were tested by using an ammonia test kit. The results of the analysis are presented in Figure 8 . This figure shows that the fresh samples change the color of the test kit. The orange color presented in the test kit shows that the sample contains $0 \%$ ammonia, which means that the fresh sample has not been degraded. Figure 8 also shows that the non-fresh samples change the indicator color to be dark green. This color change showed that non-fresh samples had ammonia contained that ranged at 10\%-20\%. This result showed that sample B did belong to the category of the non-fresh. The presence of ammonia content indicates that compounds degradation in fish products occurred. Last part of Figure 8 presents the kit results when such a sample of $\mathrm{C}$ is measured. The results show that the test paper color changed to be yellow-green. This color indicates that the sample contains an ammonia compound, indicating that some quality degradation occurs on the sample. In other words, the tested sample of $\mathrm{C}$ had a freshness level between sample A and sample B. However, the test by using the ammonia test kit had not been carried out for all samples, but only been done to randomly selected samples in order to confirm the studied samples.

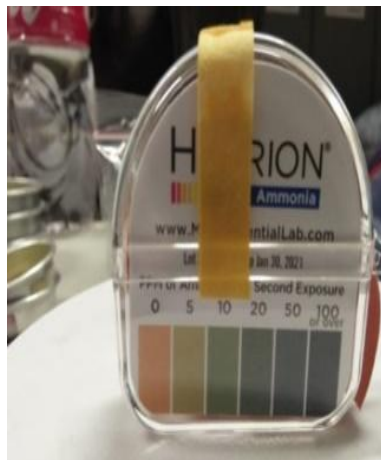

(a)

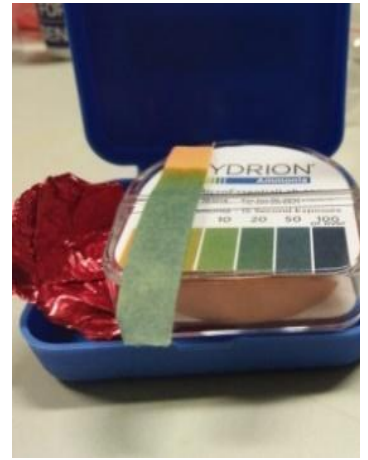

(b)

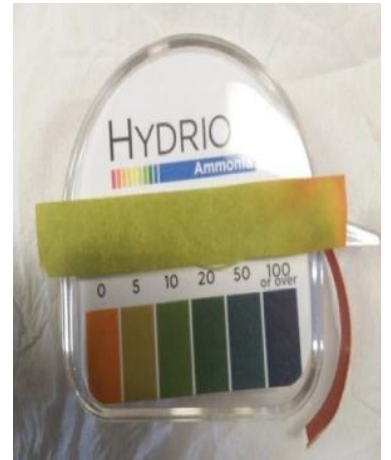

(c)

Figure 8. Ammonia test of sample; (a) fresh sample, (b) non-fresh sample, (c) sample from traditional market

\section{CONCLUSION}

Study of the determination of the freshness level of tilapia by using an e-nose has been carried out. The e-nose has been designed with a series of semiconductor gas sensors as detectors. There were three categories of tilapia samples tested in this study, fresh samples (A), non-fresh samples (B), and samples obtained randomly from traditional market (C). The collected data were formed into an aroma pattern by four pattern formation methods, namely: a) absolute data; b) normalized absolute data; c) relative data; and d) normalized relative data. Sample aroma patterns were then analyzed by using two methods, namely PCA and 
NN. The results of PCA and neural network analysis showed that the normalized absolute data method (b) provided the best classification level. The result shows that the $\mathrm{NN}$ is able to classify the fresh and non-fresh samples with the level of accuracy of $93.88 \%$. When the e-nose is applied for sample C, the result shows that $60.0 \%$ of samples matched the fresh category, $33.3 \%$ matched the non-fresh category, and $6.7 \%$ did not match both categories.

\section{ACKNOWLEDGEMENTS}

We would like to thank to Universitas Gadjah Mada (RTA Grant) for funding this research. We are also grateful to the research group of Agricultural Control \& Robotics, Department of Agricultural and Biosystems Engineering, Faculty of Agricultural Technology, Universitas Gadjah Mada (UGM).

\section{REFERENCES}

[1] H.-D. Belitz, W. Grosch, and P. Schieberle, Food Chemistry, 4th ed. Berlin Heidelberg: Springer-Verlag, 2009. doi: 10.1007/978-3-540-69934-7.

[2] Ayu Kalista, Amin Redjo, and Umi Rosidah, "Aplication of Image Processing to Determine The Freshness of Tilapia Fish (Oreochromis niloticus),” J. Pengolah. Has. Perikan. Indones., vol. 22, no. 2, Sep. 2019, doi: https://doi.org/10.17844/jphpi.v22i2.27364.

[3] A. T. S. Semeano et al., "Tilapia fish microbial spoilage monitored by a single optical gas sensor," Food Control, vol. 89, pp. 72-76, Jul. 2018, doi: 10.1016/j.foodcont.2018.01.025.

[4] I. Adoga, E. Joseph, and F. Omojowo, "Storage Life of Tilapia (Oreochromis niloticus) in Ice and Ambient Temperature," Researcher, vol. 2, no. 5, pp. 39-44, Jan. 2010.

[5] P. K. Prabhakar, S. Vatsa, P. P. Srivastav, and S. S. Pathak, "A comprehensive review on freshness of fish and assessment: Analytical methods and recent innovations," Food Res. Int., vol. 133, p. 109157, Jul. 2020, doi: 10.1016/j.foodres.2020.109157.

[6] J. Tan and J. Xu, "Applications of electronic nose (e-nose) and electronic tongue (e-tongue) in food quality-related properties determination: A review," Artif. Intell. Agric., vol. 4, pp. 104-115, Jan. 2020, doi: 10.1016/j.aiia.2020.06.003.

[7] J. Yan, R. Cui, Y. Qin, L. Li, and M. Yuan, "A pH indicator film based on chitosan and butterfly pudding extract for monitoring fish freshness," Int. J. Biol. Macromol., vol. 177, pp. 328-336, Apr. 2021, doi: 10.1016/j.ijbiomac.2021.02.137.

[8] S. Grassi, S. Benedetti, M. Opizzio, E. di Nardo, and S. Buratti, "Meat and Fish Freshness Assessment by a Portable and Simplified Electronic Nose System (Mastersense)," Sensors, vol. 19, no. 14, p. E3225, Jul. 2019, doi: $10.3390 / \mathrm{s} 19143225$.

[9] M. Falasconi, I. Concina, E. Gobbi, V. Sberveglieri, A. Pulvirenti, and G. Sberveglieri, "Electronic Nose for Microbiological Quality Control of Food Products," Int. J. Electrochem., vol. 2012, p. e715763, Feb. 2012, doi: $10.1155 / 2012 / 715763$.

[10] W. Wojnowski, T. Majchrzak, T. Dymerski, J. Gębicki, and J. Namieśnik, "Portable Electronic Nose Based on Electrochemical Sensors for Food Quality Assessment," Sensors, vol. 17, no. 12, p. E2715, Nov. 2017, doi: $10.3390 / \mathrm{s} 17122715$.

[11] B. Gunawan, S. Alfarisi, G. Satrio, A. Sudarmaji, M. Malvin, and K. Krisyarangga, "MOS gas sensor of meat freshness analysis on E-nose," TELKOMNIKA Telecommun. Comput. Electron. Control, vol. 17, no. 2, Art. no. 2 , Apr. 2019, doi: 10.12928/telkomnika.v17i2.11787.

[12] R. Sarno and D. R. Wijaya, "Recent development in electronic nose data processing for beef quality assessment," TELKOMNIKA Telecommun. Comput. Electron. Control, vol. 17, no. 1, Art. no. 1, Feb. 2019, doi: 10.12928/telkomnika.v17i1.10565.

[13] S. A. Laga and R. Sarno, "Temperature effect of electronic nose sampling for classifying mixture of beef and pork," Indones. J. Electr. Eng. Comput. Sci., vol. 19, no. 3, Art. no. 3, Sep. 2020, doi: 10.11591/ijeecs.v19.i3.pp1626-1634.

[14] Radi, S. Ciptohadijoyo, W. S. Litananda, M. Rivai, and M. H. Purnomo, "Electronic nose based on partition column integrated with gas sensor for fruit identification and classification," Comput. Electron. Agric., vol. 121, pp. 429-435, Feb. 2016, doi: 10.1016/j.compag.2015.11.013.

[15] P. Sharma et al., "Monitoring the fermentation process of black tea using QCM sensor based electronic nose," Sens. Actuators B Chem., vol. 219, pp. 146-157, Nov. 2015, doi: 10.1016/j.snb.2015.05.013.

[16] X. Lu, J. Wang, G. Lu, B. Lin, M. Chang, and W. He, "Quality level identification of West Lake Longjing green tea using electronic nose," Sens. Actuators B Chem., vol. 301, p. 127056, Dec. 2019, doi: 10.1016/j.snb.2019.127056.

[17] Radi, Muhammad Rivai, Mauridhi Hery Purnomo, D. Hardiono, and R. Kurniawan, "Discrimination of Coffee Aroma using Electronic nose based on Gas Sensor Array and Principal Component Analysis," in Jogja Int. Conf. Physic 2012, Yogyakarta, Nov. 2004, pp. 216-220.

[18] J. Rodríguez, C. Durán, and A. Reyes, "Electronic Nose for Quality Control of Colombian Coffee through the Detection of Defects in 'Cup Tests,"” Sensors, vol. 10, no. 1, pp. 36-46, Dec. 2009, doi: 10.3390/s100100036. 
[19] A. Loutfi, S. Coradeschi, G. K. Mani, P. Shankar, and J. B. B. Rayappan, "Electronic noses for food quality: A review," J. Food Eng., vol. 144, pp. 103-111, Jan. 2015, doi: 10.1016/j.jfoodeng.2014.07.019.

[20] Radi, M. Rivai, and M. H. Purnomo, "Study on Electronic-Nose-Based Quality Monitoring System for Coffee Under Roasting," J. Circuits Syst. Comput., vol. 25, no. 10, p. 1650116, Oct. 2016, doi: $10.1142 / \mathrm{S} 0218126616501164$.

[21] C. Severini, A. Derossi, A. G. Fiore, I. Ricci, and M. Marone, "The electronic nose system: study on the global aromatic profile of espresso coffee prepared with two types of coffee filter holders," Eur. Food Res. Technol., vol. 242, no. 12, pp. 2083-2091, Dec. 2016, doi: 10.1007/s00217-016-2705-1.

[22] W. Dong, J. Zhao, R. Hu, Y. Dong, and L. Tan, "Differentiation of Chinese robusta coffees according to species, using a combined electronic nose and tongue, with the aid of chemometrics," Food Chem., vol. 229, pp. 743-751, Aug. 2017, doi: 10.1016/j.foodchem.2017.02.149.

[23] P. Giungato, E. Laiola, and V. Nicolardi, "Evaluation of Industrial Roasting Degree of Coffee Beans by Using an Electronic Nose and a Stepwise Backward Selection of Predictors," Food Anal. Methods, vol. 10, no. 10, pp. 3424 3433, Oct. 2017, doi: 10.1007/s12161-017-0909-z.

[24] G. Y. F. Makimori and E. Bona, "Commercial Instant Coffee Classification Using an Electronic Nose in Tandem with the ComDim-LDA Approach," Food Anal. Methods, vol. 12, no. 5, pp. 1067-1076, May 2019, doi: 10.1007/s12161-019-01443-5.

[25] G. Marek, B. Dobrzański, T. Oniszczuk, M. Combrzyński, D. Ćwikła, and R. Rusinek, "Detection and Differentiation of Volatile Compound Profiles in Roasted Coffee Arabica Beans from Different Countries Using an Electronic Nose and GC-MS,” Sensors, vol. 20, no. 7, Art. no. 7, Jan. 2020, doi: 10.3390/s20072124.

[26] L. Capelli, S. Sironi, and R. D. Rosso, "Electronic Noses for Environmental Monitoring Applications," Sensors, vol. 14, no. 11, pp. 19979-20007, Oct. 2014, doi: 10.3390/s141119979.

[27] S. Dragonieri, G. Pennazza, P. Carratu, and O. Resta, "Electronic Nose Technology in Respiratory Diseases," Lung, vol. 195, no. 2, pp. 157-165, Apr. 2017, doi: 10.1007/s00408-017-9987-3.

[28] I. Tazi, N. L. Isnaini, M. Mutmainnah, and A. Ainur, "Principal Component Analysis (PCA) Method for Classification of Beef and Pork Aroma Based on Electronic Nose," Indones. J. Halal Res. IJHAR, vol. 1, no. 1, Art. no. 1, Feb. 2019, doi: 10.15575/ijhar.v1i1.4155.

[29] N. F. M. Radzi, A. C. Soh, A. J. Ishak, M. K. Hasan, and U. K. M. Yusof, "Aromatic Herbs Classification by using Discriminant Analysis Techniques,” Indones. J. Electr. Eng. Comput. Sci., vol. 5, no. 3, Art. no. 3, Mar. 2017, doi: 10.11591/ijeecs.v5.i3.pp530-535.

[30] O. A. Adegbola, I. A. Adeyemo, F. A. Semire, S. I. Popoola, and A. A. Atayero, "A principal component analysisbased feature dimensionality reduction scheme for content-based image retrieval system," TELKOMNIKA Telecommun. Comput. Electron. Control, vol. 18, no. 4, Art. no. 4, Aug. 2020, doi: 10.12928/telkomnika.v18i4.11176.

[31] N. Guzmán, I. Segovia, A. López, M. Ruiz-Rico, and J. Barat, "Physico-chemical and microbiological changes in commercial tilapia (Oreochromis niloticus) during cold storage," Rev. Vitae, vol. 22, Nov. 2015, doi: 10.17533/udea.vitae.v22n2a08.

[32] J. K. Heising, M. Dekker, P. V. Bartels, and M. A. J. S. van Boekel, "A non-destructive ammonium detection method as indicator for freshness for packed fish: Application on cod," J. Food Eng., vol. 110, no. 2, pp. 254-261, May 2012, doi: 10.1016/j.jfoodeng.2011.05.008.

\section{BIOGRAPHIES OF AUTHORS}
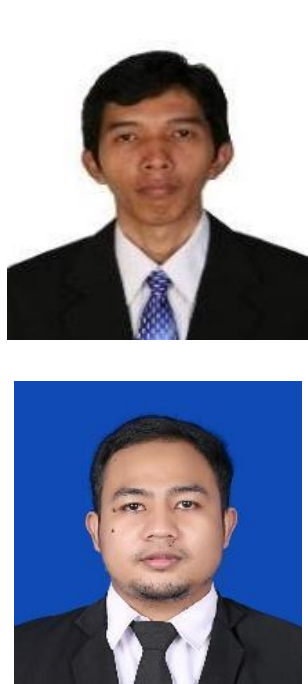

Radi, is a lecturer at the Department of Agricultural and Biosystems Engineering, Faculty of Agricultural Technology, Universitas Gadjah Mada, Indonesia. He received his bachelor's degree from that department, master's degree from Department of Electrical Engineering, Faculty of Engineering, Universitas Gadjah Mada, doctoral degree from Department of Electrical Engineering, Faculty of Industrial Technology, Institut Teknologi Sepuluh Nopember, Indonesia. Currently, he is head of Agricultural Energy \& Machinery Laboratory. His research interest is associated with the research group of agricultural control \& robotics which covers electronic nose, machine vision, bio-sensing, biosystems control \& robotics.

Eka Wahyudi is bachelor's student of Department of Agricultural and Biosystems Engineering, Faculty of Agricultural Technology, Universitas Gadjah Mada, Indonesia, Indonesia. Currently, he is a member of the research group of agricultural control \& robotics. His research interest is application of an electronic nose for food safety. 


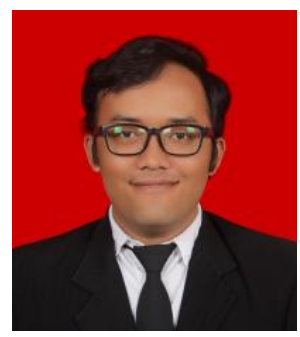

Muhammad Danu Adhityamurti is master's student of Department of Agricultural and Biosystems Engineering, Faculty of Agricultural Technology, Universitas Gadjah Mada, Indonesia, Indonesia. Currently, he is a member of the research group of agricultural control \& robotics. His research interest is application of an electronic nose for food quality process control.

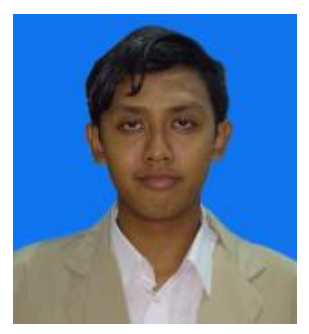

Joko Purwo Leksono Yuroto Putro is a bachelor's student of Department of Agricultural and Biosystems Engineering, Faculty of Agricultural Technology, Universitas Gadjah Mada, Indonesia, Indonesia. Currently, he is a member of the research group of agricultural control \& robotics. His research interest is application of an electronic nose for food quality assessment.

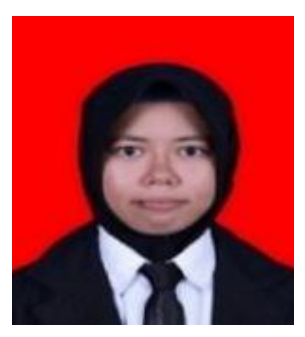

Barokah is a master's student of Department of Agricultural and Biosystems Engineering, Faculty of Agricultural Technology, Universitas Gadjah Mada, Indonesia, Indonesia. Currently, she is a member of the research group of agricultural control \& robotics. Her research interest is application of an electronic nose for food quality.

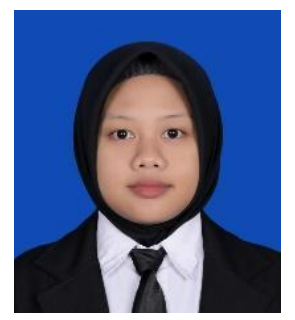

Dwi Noor Rohmah is bachelor's student of Department of Agricultural and Biosystems Engineering, Faculty of Agricultural Technology, Universitas Gadjah Mada, Indonesia, Indonesia. Currently, she is a member of She is the research group of agricultural control \& robotics. Her research interest is application of an electronic nose for food quality assessment. 